\title{
Increased salivary aldehyde dehydrogenase 1 in non-reticular oral lichen planus*
}

\author{
Arash Mansourian ${ }^{1}$ \\ Seyed Javad $\mathrm{Kia}^{3}$
}

\author{
Najmeh Shanbehzadeh ${ }^{2}$ \\ Mahdieh-Sadat Moosavi ${ }^{4}$
}

DOI: http:/ / dx.doi.org/10.1590/abd1806-4841.20174964

\begin{abstract}
BACKGROUND: Oral lichen planus is a potentially malignant disorder. One of the malignant transformation markers is cancer stem cells. One of the proposed marker for the detection of cancer stem cells's in head and neck cancer is aldehyde dehydrogenase. Recently it is shown that aldehyde dehydrogenase 1 expression in tissue samples is associated with oral lichen planus malignant transformation.

Oвјестіл: This study evaluates salivary aldehyde dehydrogenase 1 in oral lichen planus.

METHOD: Thirty patients and 30 age and sex-matched healthy volunteers were recruited. Oral lichen planus was diagnosed based on the modified World Health Organization criteria. Subjects in the case group were divided into reticular and non-reticular forms. Unstimulated salivary samples were collected at 10-12 AM. Saliva concentrations of aldehyde dehydrogenase 1 were measured by ELISA.

RESULTS: The differences between aldehyde dehydrogenase levels in the oral lichen planus group compared with the control group were not significant but aldehyde dehydrogenase in non-reticular oral lichen planus was significantly higher than that of the reticular form.

LIMITATIONS OF THE STUDY: This is a cross-sectional study, thus longitudinal studies in oral lichen planus may present similar or different results.

CONCLUSIONS: The mechanism of malignant transformation in oral lichen planus is not defined. Previous analyses revealed that the aldehyde dehydrogenase 1 expression is significantly correlated with increased risk of transformation. This finding is consistent with our results because in the erosive and ulcerative forms of oral lichen planus, which have an increased risk of transformation, salivary aldehyde dehydrogenase 1 was overexpressed. A higher salivary aldehyde dehydrogenase level in non-reticular oral lichen planus can be a defensive mechanism against higher oxidative stress in these groups. Aldehyde dehydrogenase may be one of the malignant transformation markers in oral lichen planus. Further studies are needed for introducing aldehyde dehydrogenase as a prognostic indicator in certain lesions.
\end{abstract}

Keywords: Lichen planus, oral; Aldehyde dehydrogenase; Saliva

\section{INTRODUCTION}

The World Health Organization (WHO) categorized oral lichen planus (OLP) as a "potentially malignant disorder" with undetermined malignant transformation risk. ${ }^{1}$ Lichen planus (LP) has been defined as a chronic mucocutaneous inflammatory disorder in which oral mucosa is commonly involved. It is more prevalent in white and middle-aged female patients. ${ }^{2}$ Based on recent meta-analysis, its incidence is $1.27 \%$ in the general population. ${ }^{1}$

The clinical and histological characteristics of OLP have inadequate prognostic value for malignant transformation. Great controversies exist about premalignant nature of OLP that has been the subject of several studies. ${ }^{1,3}$ One of the malignant transformation markers is cancer stem cells. They play significant role in initiation, development, recurrence, and metastasis of head and neck cancers. ${ }^{3}$ Cancer stem cells (CSCs) can be recognized and isolated from stem cells by the expression of characteristic markers. ${ }^{3}$

One of the proposed markers for the detection and isolation of CSCs in many types of cancers including head and neck cancers is aldehyde dehydrogenase (ALDH), which is a detoxifying enzyme that oxidizes intracellular aldehydes. ${ }^{4}$

Deficiency in ALDH detoxification affects the development of alcoholism, alcoholic liver diseases, and colorectal cancer. ${ }^{5}$ The reason is that acetaldehyde has straight mutagenic and carcinogenic effects and can interfere with DNA synthesis and repair. Therefore it can result in tumor development..$^{5}$ It was shown that ALDH, as

Received on 24.07.2015.

Approved by the Advisory Board and accepted for publication on 27.03.2016.

Study conducted at the Tehran University of Medical Sciences - Tehran, Iran.

Financial support: Dental Research Center / Department of Oral Medicine, School of Dentistry, Tehran University of Medical Sciences.

Conflict of interest: None.

Department of Oral Medicine, School of Dentistry, Tehran University of Medical Sciences - Tehran, Iran.

Department of Oral Medicine, School of Dentistry, Hormozgan University of Medical Sciences - Bandar Abbas, Iran.

Department of Oral Medicine, Guilan University of Medical Sciences, Dental School - Rasht, Iran.

Dental Research Center, Department of Oral Medicine, School of Dentistry, Tehran University of Medical Sciences - Tehran, Iran.

C2017 by Anais Brasileiros de Dermatologia 
a CSC marker, increases in oral squamous cell carcinoma (OSCC) and oral dysplastic lesions. ${ }^{4} \mathrm{Xu}$ et al., in 2013, hypothesized that expression of ALDH1 may be deregulated in patients with OLP. They found that ALDH1 expression was significantly associated with OLP malignant transformation. In that study ALDH1 expression was assessed in tissue samples. ${ }^{3}$

In the past decade, the number of researches for finding rapid and less invasive diagnostic tests has increased exponentially, which led to new insights in saliva as a biological fluid for clinical diagnosis. ${ }^{6}$ Saliva contains many markers from blood via passive diffusion, active transport or extracellular ultra filtration. ${ }^{7}$ Salivary analysis has two important advantages, early detection of diseases and the possibility of monitoring the disease during treatments. Also, noninvasive collection method and patient comfort during the test are other advantages. ${ }^{8,9}$ Human salivary ALDH is the body's first line of defense in the oral cavity, simultaneously with other enzymes, like glutathione S-transferases, and is known as the first barrier against toxic aldehydes in food or produced during lipid peroxidation. ${ }^{10}$

This study evaluates salivary ALDH1 as a cancer stem cell marker in OLP.

\section{MATERIALS}

\section{Subjects}

Thirty patients ( 6 men and 24 women) through convenience sampling from Oral Medicine Department and 30 healthy volunteers (4 men and 26 women), age and sex-matched, were recruited between October 2013 and February 2014.

Inclusion criteria consisted of patients with OLP that have been diagnosed clinically (presence of bilateral lesions and presence of reticular lesions elsewhere in the oral cavity) and histologically (based on the WHO modified criteria). ${ }^{11}$

As non-reticular forms of OLP have higher incidence of malignant transformations, the subjects in the case group were divided into reticular and non-reticular forms. ${ }^{12}$ Subjects with smoking habit and alcohol consumption were excluded in both groups.

Written informed consent was obtained from the patients and the study protocol was approved by the Medical Sciences Ethics committee (22964-69-02-92), which complies with the Declaration of Helsinki.

\section{Saliva collection}

The human saliva mixture varies with time because of various processes of saliva stimulation and other factors, so we have assessed unstimulated salivary samples collected at 10-12 AM and at least $2 \mathrm{~h}$ after the last intake of food or drink. ${ }^{10}$

Without any chewing movements, unstimulated saliva was obtained by expectoration, while they were in relaxed position. Saliva samples were collected in a dry plastic tube. Samples were centrifuged (2400g in $10 \mathrm{~min}$ ) and stored in plastic micro-tubes at $-20^{\circ} \mathrm{C}$ until further analysis.

\section{Measurement of salivary ALDH}

Enzyme-linked immunosorbent assay was applied to measure the saliva concentrations of ALDH1, using enzyme-linked immunosorbent assay kits from Diagnostics Biochem Canada, Inc. (Ontario, Canada). Determination of ALDH1 levels was carried out according to the manufacturers' instructions.

\section{Statistical analysis}

Statistical analysis was performed using the SPSS version 16 software. Data were expressed as mean \pm standard deviation (SD). For comparing the sex between groups, the chi-square test was used. The ANOVA test was used to determine the statistical significance of unstimulated whole saliva ALDH1 in patients and control groups, with significance defined as $\mathrm{P}<0.05$. For multiple comparisons, Tukey's post hoc test was applied.

\section{RESULTS}

There were not any significant differences in age $(P=0.469)$ and sex $(\mathrm{P}=0.176)$ between OLP and control groups. There were 24 women $(80 \%)$ and 6 men $(20 \%)$ in the OLP group and 26 women $(86.7 \%)$ and 4 men $(13.3 \%)$ in the control group. The mean age for the OLP group was $42.07 \pm 12.88$ (27-66) years and $37 \pm 7.91$ (26-51) years for the control group.

The clinical characteristics of OLP group are summarized in table 1 . In the non-reticular group 17 (80.95\%) patients had erosive lesion and 4 (19.05\%) patients had ulcerative lesion. The mean ALDH1 levels for the reticular, non-reticular and control group was $4.16 \pm 2.29,6.32 \pm 2.23$ and $6.62 \pm 2.21$, respectively. The differences between ALDH1 levels in the OLP group compare with the control group were not significant $(P=0.237)$. ALDH1 in the non-reticular OLP group was significantly higher than ALDH1 in the reticular group $(\mathrm{P}=0.019)$ (Figure 1).

\section{DISCUSSION}

Salivary ALDH1 was increased significantly in erosive and ulcerative OLP compared with the reticular ones in this study. These results may suggest ALDH1 as a prognostic marker in OLP.

\begin{tabular}{lccc} 
& \multicolumn{2}{c}{ TABLE 1: Oral lichen planus Group } & Mean Age (Range) \\
\hline Neticular & Numbers & Sex Prevalence & $42.89(27-62)$ year \\
Non-reticular & $9(30 \%)$ & $9(100 \%)$ Female & $41.71(27-66)$ year \\
(Erosive/ Ulcerative) & $21(70 \%)$ & $15(71.43 \%)$ Female & \\
& & $6(28.57 \%)$ Male &
\end{tabular}




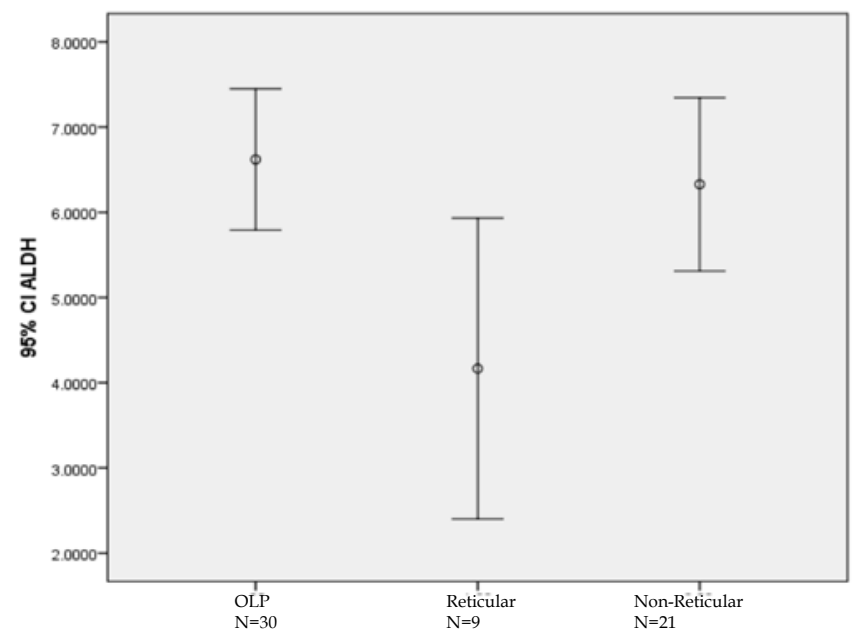

Figure 1: Graph of salivary aldehyde dehydrogenase 1 by oral lichen planus subtypes

Saliva, an ultra-filtrate of plasma, is a simple, non-invasive, efficient and cost-effective diagnostic and screening procedure. Saliva is composed of a complex mixture of secretory substances (organic and inorganic) from the salivary glands and other products resulted from the blood-derived compounds, oropharynx, upper airway, gastrointestinal reflux, gingival sulcus fluid and food deposits. ${ }^{13,14}$ Therefore we assessed ALDH1 in unstimulated saliva. Passive collection (unstimulated saliva) is the most suggested method, because most analytics may be quantified without any changes in the conventional quantification methods. ${ }^{6}$

OLP may persist many years and manifests as periods of regression and exacerbation with changes in shape, aspect and size. OLP can disappear provisionally with treatment, but their spontaneous remission is uncommon. ${ }^{2,15}$ Accordingly OLP is a long protracted chronic disease with a dynamic progression. ${ }^{15}$ The oral lesion, which is the most persistent one, may appear along with cutaneous and genital lesions or manifests solely. ${ }^{1}$

The certain etiology of the OLP is unknown, but evidences suggest the immunological process activated by an antigen that induces changes in the basal keratinocytes of the oral mucosa making them predisposed to cell immune reactions. It causes the activation of CD4+ T and CD8+ T lymphocytes and the release of cytokines, like interleukin-2 (IL-2), interferon gamma (IFN- $\gamma$ ) and tumor necrosis factor (TNF), which lead to the keratinocytes apoptosis. ${ }^{2}$

Estimates of the malignant transformation of OLP to oral squamous cell carcinoma (OSCC) ranged from 1-2\%. ${ }^{1}$ The cause of underestimated incidence or discrepancies in malignant transformation may arise from different OLP diagnostic criteria, inadequate knowledge of the post-lichen phase, poor distinguish from other keratotic and atrophic lesions, misdiagnosis of early dysplastic changes, lack of other risk factors of malignant transformation considerations (e.g., smoker), and not enough follow-ups in prospective studies..$^{15}$

The mechanism of malignant transformation in OLP is not defined. The current theory is that signals from chronic stimulation of inflammatory and stromal cells results in the imbalanced growth control of epithelial cells and along with oxidative stress, from oxidative and nitrative products, it stimulates DNA damage, which results in neoplastic events. In recent studies, OLP has been suggested to be an ideal model of inflammation induced carcinogenesis. ${ }^{1,12}$

Previous studies showed that OLP lesions other than the reticular type, which consist of the atrophic, ulcerated and erosive types of OLP, show greater incidence of malignant transformation. ${ }^{12}$ Therefore in this study we have divided OLP into reticular and non-reticular groups.

Oral cavity cancer is one of the 10 most frequently diagnosed cancers. ${ }^{16}$ Overall prognosis of oral squamous cell carcinomas is poor, with a low 5-year survival rate caused by cervical lymph node metastasis, recurrence and the absence of curative systemic therapies. ${ }^{4}$ These facts emphasize the importance of finding new markers for early detection of OSCC.

Previous analysis revealed that the ALDH1 expression was significantly correlated with increased risk of transformation. ${ }^{17}$ This finding is consistent with our result because in erosive and ulcerative forms of OLP, which have an increased risk of transformation, salivary ALDH1 was over expressed.

High ALDH activity has been suggested as a common marker for both normal and cancer stem cells. This high stem cell ALDH activity has been associated to the ALDH1A1 isozyme. ${ }^{18}$ This can explain why the measures between the OLP group and the controls are not statistically significant. The differences between normal cell ALDH and OLP cell ALDH may be illustrated by analyzing different isoforms of ALDH in future studies.

Aldehyde dehydrogenase (ALDH) enzymes have a critical role in the metabolism of numerous molecules, and in the detoxification of external and internal materials, e. g., alcohol and toxins. ${ }^{16}$ Its other functions consist of ester hydrolysis, devoting as binding proteins for various molecules, function in retinoic acid (RA) cell signaling and potentially salivary isoforms, perform as antioxidants by production of $\mathrm{NAD}(\mathrm{P}) \mathrm{H}$, scavenging hydroxyl radical and absorption of ultra violet light. ${ }^{19}$

Oxidative stress may lead to carcinogenesis due to the imbalance between the productions of reactive oxygen species (ROS) and reactive nitrogen species (RNS) and the antioxidant protection system. Increased oxidative stress and increased lipid peroxidation were observed in patients with lichen planus, especially erosive forms, which in OLP can be one of the oral cancer risk factors. ${ }^{20-22}$ Aldehyde elimination is a part of the protective mechanism against oxidative stress, because active oxygen species produce aldehyde during lipid peroxidation..$^{10}$ ALDHs decrease oxidative stress that is caused by aldehydes. ${ }^{18}$ Therefore higher salivary ALDH level in non-reticular OLP can be a defensive mechanism against higher oxidative stress in these groups.

IL-1, IL- 6 and TNF- $\alpha$ are associated with the development of oral neoplasms and they are increased in inflammatory lesions such as OLP. ${ }^{20,23}$ In recent study, it has been suggested that inflammatory lesions with production of these interleukins have a higher ALDH activity in saliva. ${ }^{20}$ Our findings are consistent with the results of all these studies, whereas erosive OLP with higher inflammation and cytokine production has an increased ALDH activity in saliva. 
There have been some concerns about using saliva as a diagnostic fluid due to salivary low concentration of analytes in comparison to blood. But this is no longer a limitation because of the advent of highly sensitive molecular methods. ${ }^{7}$ It is predictable that sensitive and specific salivary diagnostic tools with defined instructions will make salivary diagnostics practical in the near future. ${ }^{7}$

CSC such as ALDH may be a prognostic indicator for premalignant lesions. Identifying CSCs in patients and quantifying their prevalence could be used to determine the relative risk of malignant transformation in OLP.

\section{CONCLUSIONS}

In this study ALDH1 was significantly increased in the non-reticular group. It was known that ALDH is over-expressed in OSCC and growing evidence suggests that ALDH activity is a universal CSC marker. ${ }^{4,19}$ Based on our findings and previous studies that indicated that "ALDH1 overexpression in oral leukoplakia and erythroplakia may be a predictor for transformation and tumorigenicity of OSCC", we conclude that ALDH may be one of the malignant transformation markers in OLP. ${ }^{3}$ Indeed, further studies are needed in various malignant and premalignant lesions for introducing ALDH as a prognostic indicator in certain lesions.

It is recommended that follow-up of patients with dysplastic OLP should be regularly performed every two to three months. ${ }^{12}$ Based on this study, patients with dysplastic OLP who have increased level of salivary ALDH should be seen more frequently. $\square$

16. Zou B, Sun S, Qi X, Ji P. Aldehyde dehydrogenase activity is a cancer stem cell marker of tongue squamous cell carcinoma. Mol Med Rep. 2012;5:1116-20.

17. Feng JQ, Xu ZY, Shi LJ, Wu L, Liu W, Zhou ZT. Expression of cancer stem cell markers ALDH1 and Bmi1 in oral erythroplakia and the risk of oral cancer. J Oral Pathol Med. 2013;42:148-53.

18. Singh S, Brocker C, Koppaka V, Chen Y, Jackson BC, Matsumoto A, et al. Aldehyde dehydrogenases in cellular responses to oxidative/electrophilic stress. Free Radic Biol Med. 2013;56:89-101.

19. Marcato P, Dean CA, Giacomantonio CA, Lee PW. Aldehyde dehydrogenase: its role as a cancer stem cell marker comes down to the specific isoform. Cell Cycle. 2011;10:1378-84.

20. Giebułtowicz J, Wroczynski P, Samolczyk-Wanyura D. Can lower aldehyde dehydrogenase activity in saliva be a risk factor for oral cavity cancer? Oral Dis. 2013;19:763-6.

21. Shirzad A, Pouramir M, Seyedmajidi M, Jenabian N, Bijani A, Motallebnejad M Salivary total antioxidant capacity and lipid peroxidation in patients with erosive oral lichen planus. J Dent Res Dent Clin Dent Prospects. 2014;8:35-9.

22. Sezer E, Ozugurlu F, Ozyurt H, Sahin S, Etikan I. Lipid peroxidation and antioxidant status in lichen planus. Clin Exp Dermatol. 2007;32:430-4.

23. Rhodus NL, Cheng B, Myers S, Bowles W, Ho V, Ondrey F. A comparison of the pro-inflammatory, NF-KB-dependent cytokines: TNF-alpha, IL-1-alpha, IL-6 and IL-8 in different oral fluids from oral lichen planus patients. Clin Immunol. 2005; $114: 278-83$

MAILING ADDRESS:

Mahdieh-Sadat Moosavi

North Karegar St

School of Dentistry, Tehran University of Medical

Sciences

14399-55991 - Tehran,Iran.

Email:ms-moosavi@sina.tums.ac.ir

How to cite this article: Mansourian A, Shanbehzadeh N, Kia SJ, Moosavi MS. Increased salivary aldehyde dehydrogenase 1 in non-reticular oral lichen planus. An Bras Dermatol. 2017;92(1):168-71. 